University of Nebraska - Lincoln

DigitalCommons@University of Nebraska - Lincoln

1993

\title{
Determination of Radiative Transport Properties of Particle Suspensions by a Single-Scattering Experiment
}

Seung-Ki Chae

University of Minnesota

HaeOk Skarda Lee

NASA Lewis Research Center, Clewland, $\mathrm{OH}$

Follow this and additional works at: http://digitalcommons.unl.edu/nasapub

Chae, Seung-Ki and Lee, HaeOk Skarda, "Determination of Radiative Transport Properties of Particle Suspensions by a SingleScattering Experiment" (1993). NASA Publications. 171.

http://digitalcommons.unl.edu/nasapub/171

This Article is brought to you for free and open access by the National Aeronautics and Space Administration at DigitalCommons@University of Nebraska - Lincoln. It has been accepted for inclusion in NASA Publications by an authorized administrator of DigitalCommons@University of Nebraska - Lincoln. 


\title{
Determination of Radiative Transport Properties of Particle Suspensions by a Single-Scattering Experiment
}

\author{
Seung-Ki Chae* \\ Particle Technology Laboratory, Department of Mechanical Engineering, University of Minnesota, \\ Minneapolis, $M N 55455$
}

HaeOk Skarda Lee

NASA Lewis Research Center, Cleveland, OH 44135

\begin{abstract}
A light-scattering experiment on particles was performed to determine radiative transport properties of the suspension. Extinction coefficient, scattering coefficient, and phase function are the important properties. A generalized light-scattering experimental setup was designed and constructed to perform systematic research on light scattering by particles. By measuring the transmitted energy and angular scattered-energy distribution around a particle suspension, complete information on radiative transport properties could be obtained. This study focused on the performance of the
\end{abstract}

experimental setup with purely scattering particle suspensions. Transparent polymer monospheres with diameters of $0.091,0.546,1.001$, and $7.04 \mu \mathrm{m}$ were used in separate suspensions of water for this study. The radiative transport properties obtained experimentally are compared with results from Mie theory. The single-scattering condition was examined and maintained during this experiment. The number concentrations of all but the smallest particles were independently measured using an optical liquid-borne particle counter.

\section{INTRODUCTION}

Radiative transport in particle suspensions plays an important role in many engineering applications. Knowledge of the radiative transport properties of suspensions is needed to investigate the energy transfer in such participating media (Siegel and Howell, 1981). Directly, these properties help to predict and understand the multiple scattering, absorption, and emission possible within such media. Radiative transport properties can also be

This is Particle Technology Laboratory Publication No. 828 .

*'To whom correspondence should be addressed. used indirectly with light-scattering measurements to infer more fundamental properties of the particles for aerosol applications.

An experimental system called the nephelometer was designed to study the characteristics of atmospheric particulates using light scattering (Grams et al., 1975; Hansen and Evans, 1980). A commercial instrument called the Brice-Phoenix photometer was developed to measure the angular intensity of scattered light for particle suspensions (Kratohvil and Smart, 1965; Kratohvil, 1966). Another instrument called the Differential II was designed to measure the angular scattered intensity of a single, levitated particle 
(Wyatt and Phillips, 1972; Wyatt, 1980). Rapid measurement techniques were developed to obtain the distribution of angular scattered intensity for aerosol flows using an ellipsoidal mirror and a rotating aperture system (Marshall et al., 1976) or an ellipsoidal reflector and a detector array (Bartholdi et al., 1980). Experimental determination of radiative transport properties of aerosol clouds has also been investigated in the infrared range using single-scattering experiments (Love and Wheasler, 1964; Lee, 1985; Menart, 1988).

To solve the direct problem of radiative transfer in a participating medium, the following equation derived from conservation of energy must be solved (Siegel and Howell, 1981).

$$
\begin{aligned}
& \frac{\partial I(\Omega, s)}{\partial s}+\beta I(\Omega, s) \\
& =(\beta-\sigma) I_{\mathrm{b}} \\
& \quad+\frac{\sigma}{2} \int_{\Omega^{\prime}=4 \pi} P(\theta) I\left(\Omega^{\prime}, s\right) d \Omega^{\prime}
\end{aligned}
$$

where $I$ is scattered intensity in direction $\Omega, s$ is the position variable along a path, $I_{\mathrm{b}}$ is Planck function, and other variables are defined in NOMENCLATURE. As shown in Eq. 1, radiative transport properties $\beta, \sigma$, and $P(\theta)$ are needed in the analysis.

For homogeneous spherical particles with known index of refraction and size, theoretically derived transport properties can be calculated using Mie theory (Kerker, 1969; van de Hulst, 1981; Bohren and Huffman, 1983). The theoretical extinction and scattering coefficients are defined as

$$
\begin{aligned}
& \beta=\left(\pi D_{\mathrm{p}}^{2} / 4\right) N Q_{\mathrm{ext}}, \\
& \sigma=\left(\pi D_{\mathrm{p}}^{2} / 4\right) N Q_{\mathrm{sca}} .
\end{aligned}
$$

Mie scattering phase function, which represents the dimensionless distribution of angular scattered intensity, is calculated as

$P_{\mathrm{M}}(\theta)=\frac{2}{X^{2} Q_{\mathrm{sca}}}\left[i_{1}(\theta)+i_{2}(\theta)\right]$.

For more complex particle suspensions, an experimental approach is generally required to obtain radiative transport properties. This article describes a generalized experimental setup designed and calibrated using different aqueous suspensions of transparent, polymer monospheres of different sizes $\left(D_{\mathrm{p}}=0.091\right.$, $0.546,1.001$, and $7.04 \mu \mathrm{m}$ ). One application of measured radiative properties to determine the unknown refractive indices of dyed-polymer monospheres is described in the following article (Chae and Lee, 1993).

The hydrosols used in this experiment can be defined well because monodisperse particle sizes measured by electron microscope are available from the manufacturers, and number concentrations of diluted particle suspensions to satisfy the singlescattering condition are accurately measured using a liquid-borne particle counter. The experimental setup can be calibrated, because the radiative transport properties can be obtained from Mie theory as well as from this single-scattering experiment.

\section{SINGLE-SCATTERING EXPERIMENT}

A single-scattering experiment measures the scattered light from a dilute suspension of many particles. If the suspension is sufficiently dilute, the incident light beam interacts with at most a single particle in a given path. The scattered energy measured in any direction is then linearly proportional to the particle number concentration in such a medium and gives direct information on the particle characteristics required for determining the radiative transport properties. Higher number concentrations are desirable because the scattered signals increase. If the particle number concentration becomes too 
high, however, multiple scattering occurs, where a scattered beam from a particle interacts with other particles before leaving the medium (van de Hulst, 1980). The single-scattering condition also assumes independent scattering, where the scattered field from one particle is not influenced by the scattered field of an adjacent particle (Drolen, 1986).

\section{Experimental Apparatus}

The experimental apparatus was designed to measure the energy of angularly scattered and directly transmitted light. A schematic diagram of the experimental apparatus is shown in Figure 1. The figure shows a transmission or $0^{\circ}$ forwardscattering configuration. When the scattering angle increases, it is measured in counterclockwise direction. In this setup, accurate optical alignment was a major concern, in that low scattering energies had to be measured with good angular resolution. Special attention was also given to the refraction and reflection effects due to the different refractive indices of air, test cell, and aqueous suspension.

The radiation source was a randomly polarized He-Ne red laser with a wavelength of $0.6328 \mu \mathrm{m}$. The $4.0-\mathrm{mW}$ laser, with a beam diameter of $0.8 \mathrm{~mm}$ and a divergence of $1.0 \mathrm{mrad}$, is modulated at $290 \mathrm{~Hz}$ by a variable-frequency mechanical light chopper. The amplitude of the incident beam is controlled by using three neutral density filters, which are calibrated at the laser wavelength to reduce the incident beam by a combination of transmittance of $10^{-1}, 10^{-2}$, and $10^{-3}$. Controlling the incident energy with the filters made it possible to supply constant power to the photodetector during the entire angular scan for scattered-energy measurements.

Rectangular spectrophotometer cells of optical glass with path lengths of 2,5 , and $10 \mathrm{~mm}$ were used to obtain the extinction coefficient from transmission measurements. The bare laser beam was used for the scattered-energy measurements with a cylindrical quartz fluorimeter tube of 10 $\mathrm{mm}$ inner diameter and $12 \mathrm{~mm}$ outer diameter.

The test cells for the scattering or transmission measurements were located at the center of the rotator shown in Figure 1; they were supported by a $X-Y$ micropositioner and a tilt angle control device. The supporting equipment was fixed on the optical table and did not rotate with the detection optics. The tilt of a test cell was adjusted until the reflected beams from both sides were nearly matched as one point at the backside of the incident aperture. The tilt angle was especially important for the rectangular cells, because it aggravated the problem of reflection losses owing to the front and back sides of the rectangular cells not being perfectly parallel. For the scattering measurements, the centerline of the cylindrical cell had to be aligned with the line of rotation. A second $\mathrm{He}-\mathrm{Ne}$ laser aimed at the test cell $90^{\circ}$ from the incident beam was used to help in the initial positioning of the test cell.

Even with a careful alignment of the cylindrical test cell, the curvature of the cell introduced extraneous radiation that interfered with the scattered-radiation measurements. The cylindrical quartz cell $\left(m_{\mathrm{g}}=1.458\right)$ filled with water $\left(m_{\mathrm{w}}=1.332\right)$ acted as a large lens to focus beam on a point just beyond the cylinder. The directly transmitted beam spread out after the focal point and was greater than the forward-scattering component in the region near $0^{\circ}$. In the backward-scattering region near $180^{\circ}$, the incident beam was directly reflected from the cylinder's outer surface. These affected regions in the forward and backward directions are reduced if the incident beam size is small. This is the reason for using the bare laser beam in the scattering experiment. With the 


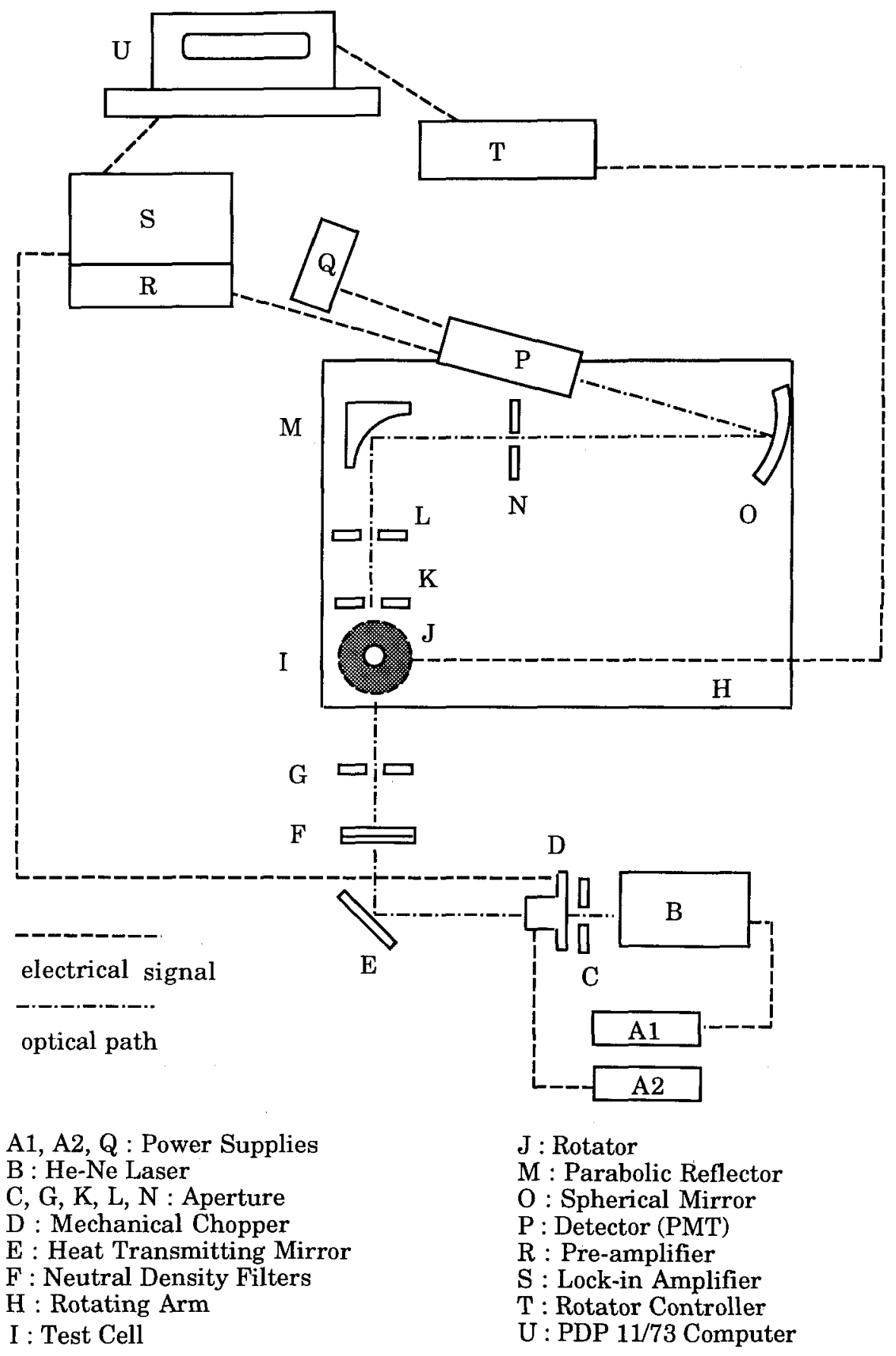

FIGURE 1. Schematic diagram of the light-scattering experimental apparatus. 


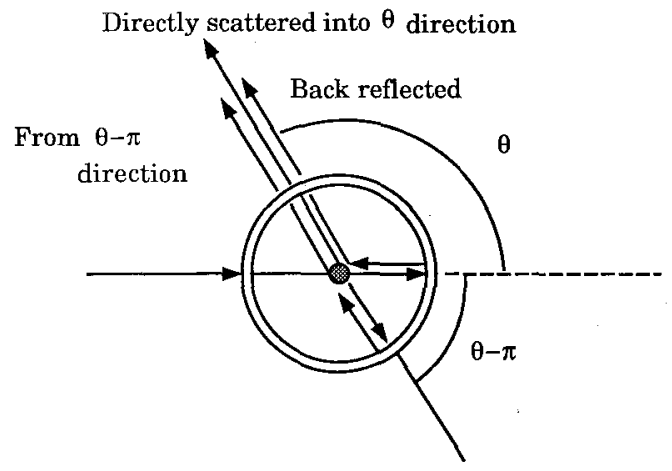

FIGURE 2. The effects of test cell on the angular scattered energy measurement.

bare laser beam, the transmission-affected region was at least from $0^{\circ}$ to $4^{\circ}$, and the reflection-affected region was from $154^{\circ}$ to $180^{\circ}$. Only the data from $4^{\circ}$ to $154^{\circ}$ can be used to determine the particulate scattering properties.

In the usable region of $4^{\circ}$ to $154^{\circ}$, multiple internal reflections caused the angular energy from the clean water to be higher than that from the particle suspension. When a particle scatters light in a $\theta$ direction in this region, two other important components besides the directly scattered component are present because of the multiple internal reflections. Three main components included in a $\theta$ direction are shown in Figure 2. One component is from the back reflected beam at the inner surface. This reflected beam will again be incident on the same particle and cause scattering into the $\theta$ direction. The other component is from the beam scattered in the $\theta-\pi$ direction by the particulate that is reflected at the inner cylinder and transmitted into the $\theta$ direction. The magnitude of these two components is same, and it can be several times bigger than the directly scattered component in the $\theta$ direction for highly forward-scattering particles.

A data reduction method to include the internal reflection effect, suggested by
Kratohvil (1966) for a Brice-Phoenix photometer cell, could not be applied for this experiment, in that a Brice-Phoenix cell has two flat sides that prevent the incident laser beam from being focused by the cylindrical cell. Drolen et al. (1987) attempted to remove the internal reflection effect by tilting the test cell for backscattered energy measurements. In this study, the internal multiple reflection effect is simply removed by inserting a stainless steel tube with a partial horizontal slit, which is coated using a black, solar-absorber.

Figure 3 shows a schematic top view of the cylindrical cell with the black tube. The directly transmitted beam was absorbed, which eliminated the secondary scattering by the particles in the $\theta$ direction. The tube also absorbed the scattered beam in the $\theta-\pi$ direction. The removal of the strong internal cylinder reflections made it possible to measure the details of the angular distribution of scattered energy. Because of the light trap for the transmitted component, measurements of scattered radiation ranged from $12^{\circ}$ to $154^{\circ}$.

The detection equipments were located on a rotating arm moved by a computercontrolled stepping motor with an angular resolution of $0.01^{\circ}$. The detector assembly

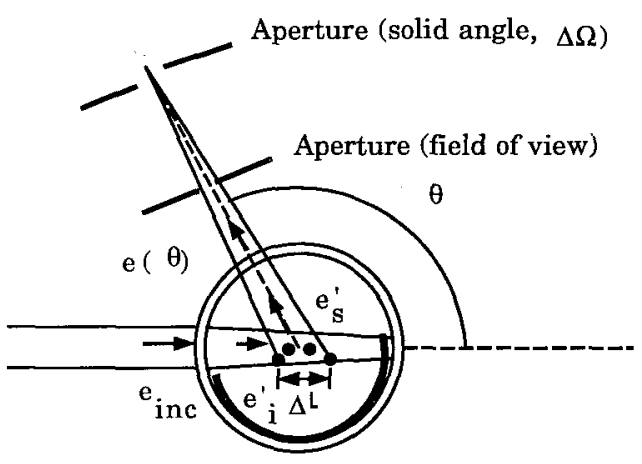

FIGURE 3. Schematic of the viewing volume inside the test cell with the black tube. 
could be rotated in counterclockwise direction from $-10^{\circ}$ to $170^{\circ}$ without being blocked by other optical components. The scattered or transmitted light beam passed through two adjustable circular apertures; the first one of $2 \mathrm{~mm}$ in diameter set the field of view, and the next one of $1 \mathrm{~mm}$ in diameter defined the detection solid angle. The solid angle for detection was $4.0 \times 10^{-5}$ steradian. Scattered light was focused by a $90^{\circ}$ off-axis paraboloidal reflector with a focal length of $33 \mathrm{~mm}$. A concave, spherical mirror with a $50-\mathrm{mm}$ focal length was used to redirect and focus the beam to the detector. An end-on type photomultiplier tube (PMT) is used for detection. A constant $800-\mathrm{V}$ was supplied from a high-voltage DC power supply.

The current signal produced by the PMT was converted into voltage and amplified by a current preamplifier. A lock-in amplifier was used to select the appropriate radiation component based on the frequency of the light chopper. This eliminated most of the stray radiation in the measurement of light signals at a very low level. Data acquisition was performed by using a PDP 11/73 microcomputer (Digital Equipment Corp.; Marlboro, MA), which also handled the programmable stepper motor controller.

All the optical components on were on a standard optical table with non-isolating legs, and they were covered with a black enclosure to eliminate background noise and to protect the PMT from excess current. Although not drawn in Figure 1, radiation shields covered the detection equipments and were placed around the incident beam.

\section{Test Particle Suspensions}

Four different sizes of the transparent, polymer monosphere suspensions made by Seradyn Inc., Indianapolis, IN $\left(D_{\mathrm{p}}=0.091\right.$, $0.546 \mu \mathrm{m})$ and Duke Scientific Corp., Palo
Alto, CA $\left(D_{\mathrm{p}}=1.001,7.04 \mu \mathrm{m}\right)$ were used for this study. The manufacturer's particle sizes determined by electron microscope showed fairly good monodisperse characteristics with the ratio of standard deviation to mean size below 1.5\% (Bangs, 1987).

The number concentrations must be adequately controlled to ensure singlescattering conditions for the experiment. The radiative properties described in Eqs. 2 and 3 also require the number concentration information. Based on the initial number concentration given by the manufacturer, the suspensions were diluted to a desired concentration. For the dilution, 18 $M \Omega$ clean water is used. An ultrasonic bath was used for both cleaning test cells and stirring the particle suspensions to achieve uniform number concentrations.

Finally, the number concentrations of the dilute polymer monosphere suspensions were measured by using an optical liquid-borne particle counter (PMS Inc., Boulder, CO; LLPS-X with IMOLV-.3) with a range of detectable particle size from 0.3 to $15 \mu \mathrm{m}$. The experimental setup for this liquid-borne particle-sizing and counting system was described by Chae (1991). If the particle size is above $0.5 \mu \mathrm{m}$ and the number concentration is between $5.0 \times 10^{2}$ and $1.0 \times 10^{7} 1 / \mathrm{cm}^{3}$, the counting efficiency error is $<1 \%$ (Grant, 1988).

\section{RESULTS}

Radiative transport properties can be calculated using Eqs. 2-4 from Mie theory and be obtained from the measurements of the angular-light energy. The extinction coefficient is obtained from measurements of transmitted energy, $e\left(0^{\circ}\right)$, using the rectangular spectrophotometer cell. The scattering coefficient and phase function are derived from measurements of angular scattered-energy, $e(\theta)$, using the cylindrical quartz cell. The single-scatter- 
ing requirement is discussed in connection with the extinction coefficient and phase function.

\section{Extinction Coefficient}

The extinction coefficient of a singlescattering particulate suspension can be found from Bouguer's law:

$$
\frac{I\left(0^{\circ}\right)}{I_{\text {inc }}}=\exp (-\beta L) \text {. }
$$

Transmissivity measurements of different small particles in water, such as carbon, tungsten, aluminum oxide, and hafnium carbide, were performed by Lanzo and Ragsdale (1962), and they experimentally confirmed Bouguer's law.

The experimental extinction coefficient including the multiple reflections inside the rectangular spectrophotometer cell is expressed as follows (Drolen et al., 1987):

$\beta_{\mathrm{E}}=$

$$
-\frac{1}{L} \ln \left[\frac{-\left(1-\rho_{\mathrm{o}}\right)^{2}+\sqrt{\left(1-\rho_{\mathrm{o}}\right)^{4}+4 T^{2} \rho_{\mathrm{o}}^{2}}}{2 T \rho_{\mathrm{o}}^{2}}\right]
$$

where the measured transmittance $T$ is

$T=\frac{e\left(0^{\circ}\right)}{e_{\text {inc }}}$,

and the combined normal reflectance is obtained from Fresnel relations as

$$
\begin{aligned}
& \rho_{\mathrm{o}}=\frac{\rho_{1}+\rho_{2}-2 \rho_{1} \rho_{2}}{1-\rho_{1} \rho_{2}}, \\
& \rho_{1}=\left[\frac{m_{\mathrm{g}}-1}{m_{\mathrm{g}}+1}\right]^{2}, \quad \rho_{2}=\left[\frac{m_{\mathrm{w}}-m_{\mathrm{g}}}{m_{\mathrm{g}}+m_{\mathrm{w}}}\right]^{2} .
\end{aligned}
$$

In Eq. $6 \mathrm{c}, \rho_{1}$ and $\rho_{2}$ are the normal reflectance between air and optical glass, and optical glass and water, respectively.
The refractive indices are taken to be $m_{\mathrm{g}}=1.55$ and $m_{\mathrm{w}}=1.332$ at a wavelength of $0.6328 \mu \mathrm{m}$. The calculated $\rho_{\mathrm{o}}$ is 0.05183 , while a value from transmission measurement with clean water is 0.04343 . Consequently the experimental refractive index of optical glass is $m_{\mathrm{g}}=1.51$ with the water refractive index $m_{\mathrm{w}}=1.332$. In Eq. $6 \mathrm{~b}, e\left(0^{\circ}\right)$ is the transmitted energy measured at $0^{\circ}$, and $e_{\text {inc }}$ is the incident energy measured without the test cell in direction shown in Figure 1. In Eq. 6a, L is the inner width of the test cell.

The measured transmittances (Eq. 6b) of six polymer monosphere suspensions $\left(D_{\mathrm{p}}=1.001 \mu \mathrm{m}\right)$ are displayed in Table 1. Five measurements at different locations of the 10-mm-thick, rectangular spectrophotometer cell are averaged to obtain the transmittances. If there are no strong multiple-scattering effects, the transmittance should show a logarithmically linear, decreasing relationship with the number concentration.

The transmittance measurements shown in Figure 4 indicate no significant multiple scattering. The figure shows the good comparison between the calculated single-scattering transmittance and the experimental transmittances for a polymer monosphere suspension with $D_{\mathrm{p}}=$ $1.001 \mu \mathrm{m}$. The expression for the calculated transmittance is given below, and it includes Fresnel reflections at the rectangular test cell.

$$
T=\frac{\left(1-\rho_{\mathrm{o}}\right)^{2} e^{-\tau}}{1-\rho_{\mathrm{o}}^{2} e^{-2 \tau}} \text {. }
$$

TABLE 1. Experimental Transmission Results

\begin{tabular}{lccc}
\hline & $T$ & $N($ no. $/ \mathrm{ml})$ & $\beta_{\mathrm{E}}(1 / \mathrm{cm})$ \\
\hline Transparent & 0.8709 & $0.251 * 10^{7}$ & 0.0511 \\
PSL & 0.8011 & $0.502 * 10^{7}$ & 0.134 \\
$\left(D_{\mathrm{p}}=1.001 \mu \mathrm{m}\right)$ & 0.7234 & $1.044 * 10^{7}$ & 0.236 \\
& 0.5494 & $2.458 * 10^{7}$ & 0.511 \\
& 0.4543 & $3.480 * 10^{7}$ & 0.701 \\
& 0.3284 & $5.136 * 10^{7}$ & 1.025 \\
\hline
\end{tabular}




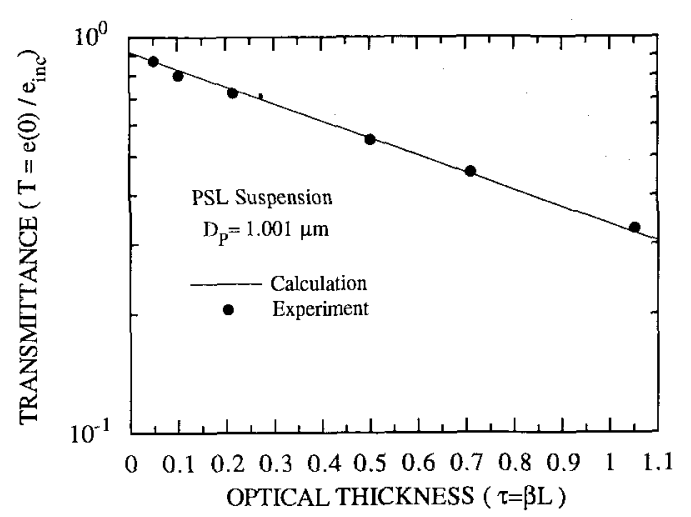

FIGURE 4. Transmittance of polymer monosphere $\left(D_{\mathrm{p}}=1.001 \mu \mathrm{m}\right)$ suspension.

In addition to the criterion that Bouguer's law (Eq. 5) should be valid for a medium as the number concentration changes, other single-scattering criteria have been reported. An optical thickness of $<0.1$ is recommended by van de Hulst (1981). Experimentally the logarithmic, inverse transmittance $\left\{\ln \left[e_{\text {inc }} / e\left(0^{\circ}\right)\right]\right\}$ has to be <3.0, and the half angle of reception must be $<1^{\circ}$ (Boll and Sliepcevich, 1956). The half angle of reception for this experimental setup is $0.72^{\circ}$, and $\left\{\ln \left[e_{\text {inc }} / e\left(0^{\circ}\right)\right]\right\}$ is $<1.5$. The optical thickness is between 0.05 and 1.05 , which is not totally below the 0.1 value recommended by van de Hulst (1981). However, Menart et al. (1989) point out that the actual optical depth for scattering is usually smaller than that which is based on the overall diameter of the test section.

The accuracy of the transmittance measurements are assessed by comparing the measured results with Mie theory. The comparisons between theoretical and experimental extinction efficiency are shown in Table 2. The results from the suspension with $0.091-\mu \mathrm{m}$ diameter polymer monospheres is not shown in the extinction coefficient table, because this particle diameter is below the detectable size limit ( $\left.D_{\mathrm{p}}=0.3 \mu \mathrm{m}\right)$ of the optical liquid-borne particle counter in this experiment. Differences between $Q_{\text {ext, M }}$ and $Q_{\text {ext, E }}$ were caused by the experimental uncertainties that come mainly from variations in measured number concentration $(N)$, transmitted energy $\left[e\left(0^{\circ}\right)\right]$ at different locations of the cell, and incident laser energy $\left(e_{\mathrm{inc}}\right)$.

\section{Phase Function}

Physically the scattering phase function compares the actual angular intensity distribution to an equivalent isotropic intensity (Siegel and Howell, 1981). The experimental phase function is obtained using the angular scattered energy $e(\theta)$ from experiment as follows;

$$
\begin{aligned}
P_{\mathrm{E}}(\theta) & =\frac{I(\theta)}{\frac{1}{4 \pi} \int_{0}^{2 \pi} \int_{0}^{\pi} I(\theta) \sin \theta \mathrm{d} \theta \mathrm{d} \varphi} \\
& =\frac{e(\theta)}{\frac{1}{2} \int_{0}^{\pi} e(\theta) \sin \theta \mathrm{d} \theta} .
\end{aligned}
$$

To obtain the scattered energy from the particle suspension, the background

TABLE 2. Theoretical and Experimental Extinction Efficiencies

\begin{tabular}{ccccccr}
\hline$D_{\mathrm{p}}(\mu \mathrm{m})$ & $T$ & $\beta_{\mathrm{E}}(1 / \mathrm{cm})$ & $N(\mathrm{no} . / \mathrm{ml})$ & $Q_{\mathrm{ext}, \mathrm{E}}$ & $Q_{\mathrm{ext}, \mathrm{M}}$ & $\Delta \%$ \\
\hline 0.546 & 0.584 & 0.45 & $2.781 * 10^{8}$ & 0.691 & 0.911 & -24.1 \\
1.001 & 0.549 & 0.512 & $2.458 * 10^{7}$ & 2.647 & 2.572 & 2.9 \\
7.04 & 0.458 & 0.693 & $7.06 * 10^{5}$ & 2.522 & 2.354 & 7.1 \\
\hline
\end{tabular}


angular energy was measured and subtracted with just the clean water in the test cell while the black tube is inside. The background light signal was of an $10^{1}$ to $10^{3}$ order of magnitude smaller than that from the polymer monosphere suspension in the side scattering region. Measurements near the transmission-affected region $\left(0^{\circ}\right.$ to $\left.10^{\circ}\right)$ and the outer tube-reflection regions $\left(154^{\circ}\right.$ to $\left.170^{\circ}\right)$ were discarded, since the background angular energy was bigger or was of nearly the same order of magnitude as the scattered energy from particle suspensions. No data was taken in the region from $170^{\circ}$ to $180^{\circ}$ because of physical obstructions.

The experimental phase function derived in Eq. 8 requires integration of the scattered energy in the whole angular region from $0^{\circ}$ to $180^{\circ}$. Extrapolations in the forward $\left(0^{\circ}\right.$ to $\left.10^{\circ}\right)$ and backward $\left(154^{\circ}\right.$ to $180^{\circ}$ ) scattering regions are needed to obtain the experimental phase function. For most particles, a linear extrapolation is acceptable in the backward region due to the relatively small and smooth variations. For large particles, however, relatively strong variations can be expected in the forward scattering region, and linear extrapolation is often inadequate. An extrapolation is always needed in the $0^{\circ}$ direction, since an experimental distinction between the transmitted and forward-scattered energy in this direction is impossible.

If a reasonable estimate of a particle size parameter and relative refractive index $\left(m_{\mathrm{p}} / m_{\mathrm{w}}\right)$, are available, Mie phase function results can be used to improve the extrapolation procedure (Menart et al., 1989). Menart et al. (1989) suggested an extrapolation technique taking an average value of the available ratio of experimental data to Mie phase function data $\left[e(\theta) / P_{M}(\theta)\right]$ in a wide angular region. This study used slope extrapolation based on Mie phase function and experimental data in a forward scattering re- gion. The current extrapolation can be expressed as

$$
\begin{aligned}
& \frac{e\left(\theta_{i}\right)-e\left(\theta_{i+1}\right)}{e\left(\theta_{i+1}\right)-e\left(\theta_{i+2}\right)} \\
& \quad=\frac{P_{\mathrm{M}}\left(\theta_{i}\right)-P_{\mathrm{M}}\left(\theta_{i+1}\right)}{P_{\mathrm{M}}\left(\theta_{i+1}\right)-P_{\mathrm{M}}\left(\theta_{i+2}\right)}
\end{aligned}
$$

where $i$ starts at angular point of $10^{\circ}$ and decreases to $0^{\circ}$ in a $2^{\circ}$ interval. Experimental data are available from $12^{\circ}$. This technique is especially good for estimating the light scattering by large particles.

No viewing volume correction is applied for this experiment, although a $\sin \theta$ correction is traditionally applied to account for the changing viewing volume with angle (Kratohvil et al., 1965; Holland, 1980; Wagner, 1985). The $\sin \theta$ correction is theoretically derived for illuminated regions with simple shapes and can be shown to be an important calibration factor for such systems. The shape of the illuminated region is more complex for the current experimental setup, and a simple correction factor cannot be derived. Instead, since this correction is purely geometric in nature and must apply equally to all types of particles, calibrations with the transparent, monosphere suspensions were performed. Figure 5 shows the comparison of experimental and Mie phase functions for $D_{\mathrm{p}}=1.001$ $\mu \mathrm{m}$. Such calibrations clearly showed that a constant viewing volume without any angle related correction factors gives a better comparison with the theoretical results than with the traditional $\sin \theta$ correction.

The single-scattering condition is further examined for the suspensions used to determine the phase functions. One simple way to determine the single-scattering limit is to show that a change in the number concentration results in a linear change in the scattered energy. The increases in scattered energy at fixed angles 


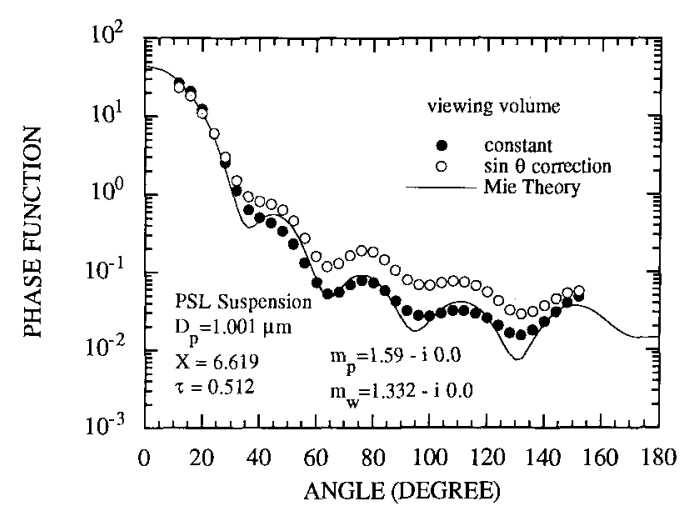

FIGURE 5. Effect of viewing volume correction for polymer monosphere $\left(D_{\mathrm{p}}=1.001 \mu \mathrm{m}\right)$ suspension.

$\left(30^{\circ}, 40^{\circ}, 60^{\circ}\right)$, shown in Figure 6 , are reasonably linear as the optical thickness increases. This verifies the single-scattering condition for most of the optical thicknesses considered.

The comparisons of Mie and extrapolated experimental phase functions are shown in Figures 7-9 for the different size transparent, polymer-monosphere suspensions. The dotted lines show the forward and backward extrapolations using Eq. 9. For test suspensions, experimental optical thicknesses used are 0.45

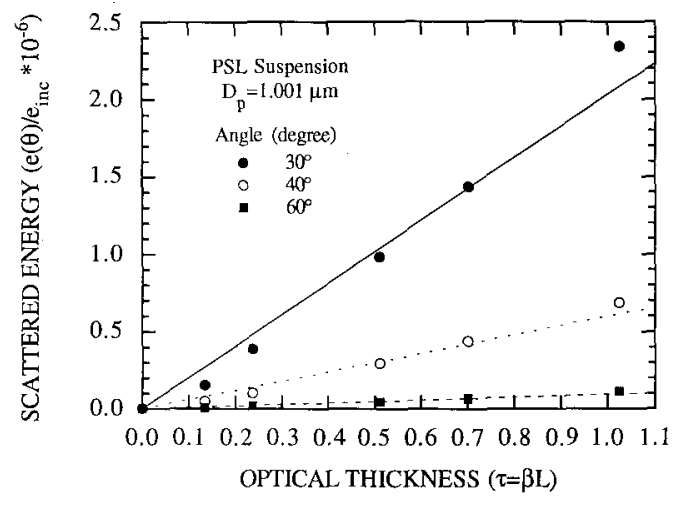

FIGURE 6. Scattered energy at fixed angles for polymer monosphere $\left(D_{\mathrm{p}}=1.001 \mu \mathrm{m}\right)$ suspensions.

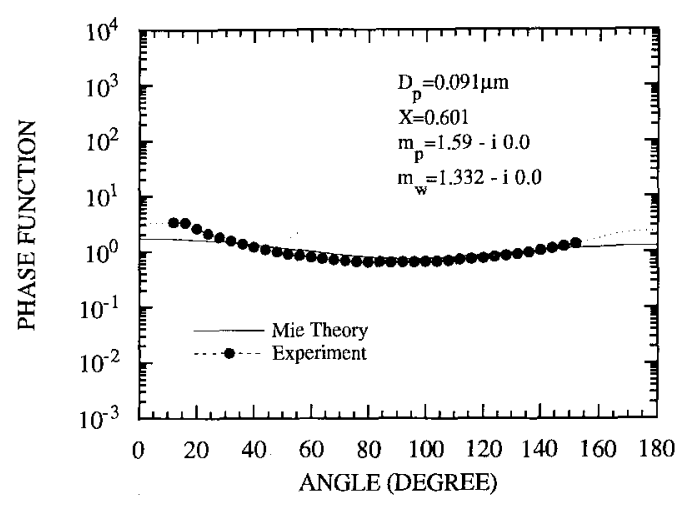

FIGURE 7. Phase function of polymer monosphere $\left(D_{\mathrm{p}}=0.091 \mu \mathrm{m}\right)$ suspension.

for $D_{\mathrm{p}}=0.546 \mu \mathrm{m}$, and 0.693 for $D_{\mathrm{p}}=$ $7.04 \mu \mathrm{m}$. Based on the prepared dilution ratio, the optical thickness for $D_{\mathrm{p}}=0.091$ $\mu \mathrm{m}$ is calculated to be 0.5 .

Figure 7 shows that the phase function is nearly isotropic at a small particle size parameter (Rayleigh scattering). As the particles become bigger, phase functions become more anisotropic with some pronounced peaks as in Figure 8 (Mie scattering). Figure 9 shows high forward scattering with many peaks at a larger $X$ (geometric scattering). Primarily because of the finite size of receiving aperture,

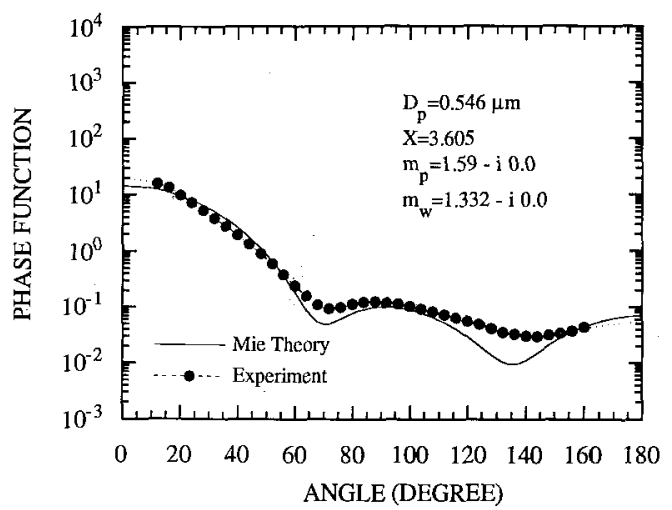

FIGURE 8. Phase function of polymer monosphere $\left(D_{\mathrm{p}}=0.546 \mu \mathrm{m}\right)$ suspension. 


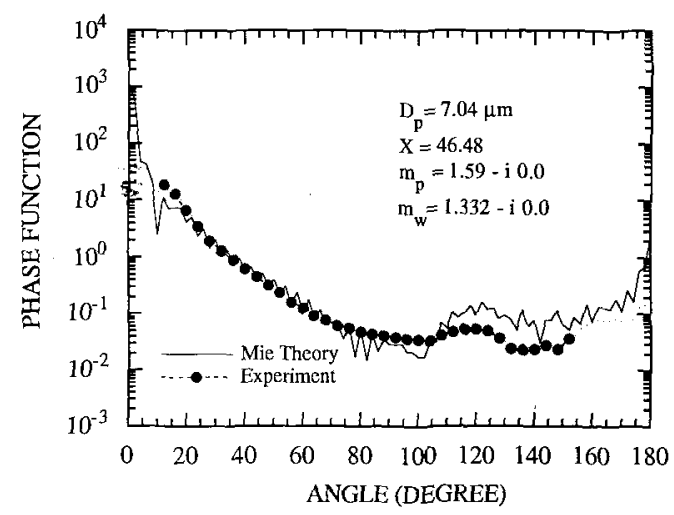

FIGURE 9. Phase function of polymer monosphere $\left(D_{\mathrm{p}}=7.04 \mu \mathrm{m}\right)$ suspension.

angular averaging effects which remove the strong oscillations in the phase function can be seen for $D_{\mathrm{p}}=7.04 \mu \mathrm{m}$. The experimental phase functions agree well with and distinguish the pronounced peaks in Mie phase functions despite low light signals. Effects of polarization, particle size discrepancy, and the incident aperture size was investigated by Kratohvil et al. (1965) and were shown to be very small compared to the effects already discussed.

\section{Asymmetry Factor}

The experimental asymmetry factor, which is a useful parameter to describe a phase function, can be calculated by integrating the experimental phase function;

$$
\langle\cos \theta\rangle=\frac{1}{2} \int_{-1}^{1} P(\theta) \cos \theta \mathrm{d}(\cos \theta) \text {. }
$$

The comparisons between experimental and theoretical asymmetry factors are displayed in Table 3. Because the asymmetry factor weighs the forward scattering region more heavily, a good extrapolation technique is especially important for this property. The extrapolation based on Mie phase function slopes (Eq. 9) provides a better asymmetry factor than the method
TABLE 3. Theoretical and Experimental Asymmetry Factors

\begin{tabular}{cccc}
\hline$D_{\mathrm{p}}(\mu \mathrm{m})$ & $\langle\cos \theta\rangle_{,}$ & $\langle\cos \theta\rangle_{M}$ & $\%$ difference \\
\hline 0.091 & 0.0764 & 0.0626 & 22.0 \\
0.546 & 0.837 & 0.842 & -0.59 \\
1.001 & 0.912 & 0.918 & -0.65 \\
7.04 & 0.921 & 0.906 & 1.65 \\
\hline
\end{tabular}

based on Mie phase function averages or linear extrapolation. The $\%$ difference in $\langle\cos \theta\rangle$ is large only for $D_{\mathrm{p}}=0.091 \mu \mathrm{m}$, and this comes from the remaining transmission-affected error in the forward scattering region shown in Figure 7. The agreement is good for the larger particles.

\section{Scattering Coefficient}

The scattering coefficient is perhaps the most difficult property to determine experimentally. It can be derived based on the definition using the phase function (Eq. 13-45, Siegel and Howell, 1981), and the incident and scattered energies at the viewing volume, shown in Figure 3 as $e_{i}^{\prime}$ and $e_{s}^{\prime}$. The scattered intensity is described in this system as

$I_{\mathrm{s}}^{\prime}(\theta)=\frac{e_{\mathrm{s}}^{\prime}(\theta)}{\Delta \Omega \Delta A \Delta \Omega_{\mathrm{i}} \Delta \lambda}$.

The incident intensity is expressed using the incident energy to the viewing volume in Figure 3 as

$I_{\mathrm{i}}^{\prime}=\frac{e_{\mathrm{i}}^{\prime}}{\Delta \Omega_{\mathrm{i}} \Delta A \Delta \lambda}$.

In these equations, $\Delta \Omega$ is the scattering solid angle shown in Figure $3, \Delta A$ is the cross-sectional area of the viewing volume, $\Delta \Omega_{\mathrm{i}}$ is the incident-beam solid angle, and $\Delta \lambda$ is the wavelength interval.

Since the measured incident and scattered energy are $e_{\text {inc }}$ and $e(\theta)$, the scattering coefficient should be expressed using measured values instead of $e_{i}^{\prime}$ and $e_{s}^{\prime}$. The scattering coefficient is described be- 
low using the participating medium length $\Delta L$ shown in Figure 3.

$$
\begin{aligned}
\sigma= & \frac{4 \pi I_{\mathrm{s}}^{\prime}(\theta)}{I_{\mathrm{i}}^{\prime} \Delta L P(\theta)} \\
= & \frac{2 \pi \int_{0}^{\pi} e(\theta) \sin \theta \mathrm{d} \theta}{e_{\mathrm{inc}}} \\
& \times \frac{1}{\Delta \Omega} \frac{1}{\Delta L(\theta)} K(\beta, \theta)
\end{aligned}
$$

with a correction factor $K(\beta, \theta)$ that incorporates the attenuation effects of the incident and scattered beams. As the scattering coefficient is a property which should be independent of direction, the angle dependent terms can be combined into one calibration factor $G(\beta)$;

$G(\beta)=\frac{K(\beta, \theta)}{\Delta L(\theta)}$.

This calibration factor was experimentally determined by using transparent polymer monosphere suspensions. Table 4 displays the experimental calibration factors for the different particle suspensions. As the number concentration of $D_{\mathrm{p}}=$ $0.091 \mu \mathrm{m}$ can not be accurately measured by using an optical liquid-borne particle counter, the calibration factor for this size might have larger errors compared to other suspensions. For the transparent monospheres considered, the calibration factors ensure that the extinction and scattering coefficients are equal to each other.

The calibration factor $G(\beta)$ is expected to only be a function of the extinction coefficient for a given experimental setup, since it comes from the attenuation effects of particles outside the viewing volume. It is then reasonable to assume that the calibration factors in Table 4 would be directly applicable to absorbing particles, as long as the actual and calibration suspensions have the same extinc-
TABLE 4. Experimental Calibration Factor for Scattering Coefficient

\begin{tabular}{cccc}
\hline$D_{\mathrm{p}}(\mu \mathrm{m})$ & $\beta_{\mathrm{E}}(1 / \mathrm{cm})$ & $G(\beta)$ & $\sigma_{\mathrm{E}}(1 / \mathrm{cm})$ \\
\hline 0.091 & 0.5 & 7.41 & 0.5 \\
0.546 & 0.45 & 5.18 & 0.45 \\
1.001 & 0.512 & 4.98 & 0.512 \\
7.04 & 0.693 & 8.42 & 0.693 \\
\hline
\end{tabular}

tion coefficient. Unfortunately, owing to a lack of well-characterized absorbing monospheres, it was not possible to verify this assumption in the current study.

\section{CONCLUSIONS}

Radiative transport properties of particle suspensions were determined by a singlescattering experiment and compared with Mie theory. The experimental apparatus was designed, and a calibration study was performed by measuring the transmission and distribution of angular scattered-energy from polymer monosphere suspensions of different sizes ( $D_{\mathrm{p}}=0.091,0.546$, 1.001 , and $7.04 \mu \mathrm{m})$. Each number concentration of diluted suspension was independently measured using an optical liquid-borne particle counter, except for $D_{\mathrm{p}}$ $=0.091 \mu \mathrm{m}$ which is smaller than the lower detection limit of the counter.

Measurements were taken using particle suspensions in the optical thickness range from 0.05 to 1.0 . The single-scattering condition was shown to be valid in this range by transmission measurements using rectangular spectrometer cells. Scattered energy measurements at fixed angles also verified the single-scattering condition.

The comparisons of the experimental and theoretical extinction coefficients show some discrepancy and may include large errors from the uncertainty of measurement. A new calibration factor for determining the scattering coefficient is introduced. The advantage of this calibration factor is that it could be experimen- 
tally determined for transparent particle suspensions.

The scattering coefficient, phase function, and asymmetry factor all required the full angular scattered energy information from $0^{\circ}$ to $180^{\circ}$. Because of the unavoidable physical difficulties in measuring the entire scattered field, extrapolation was needed in forward scattering region $\left(0^{\circ}\right.$ to $\left.12^{\circ}\right)$ and backward scattering region $\left(154^{\circ}\right.$ to $\left.180^{\circ}\right)$. As the extrapolated data sometimes have significant effects on the experimental radiative properties, special cautions should be taken. The proposed slope extrapolations based on Mie theory gave very accurate phase function and asymmetry factor results, but these extrapolation calculations require information on the particle refractive index. A technique for determining the unknown refractive indices of a particle using this single-scattering experimental setup is presented in the following paper (Chae and Lee, 1993).

This research was supported in part by NSF/CBT 8451076 and by a grant to the University of Minnesota Particle Technology Laboratory by the Particulate Contamination Control Research Consortium. Members of the consortium include Air Product and Chemicals, Inc., Airco/The BOC Group, Applied Materials, FSI International, GM/Delco Electronics, IBM Corporation, Imprints, Millipore Corporation, Nupro Company, and TSI, Inc.

\section{NOMENCLATURE}

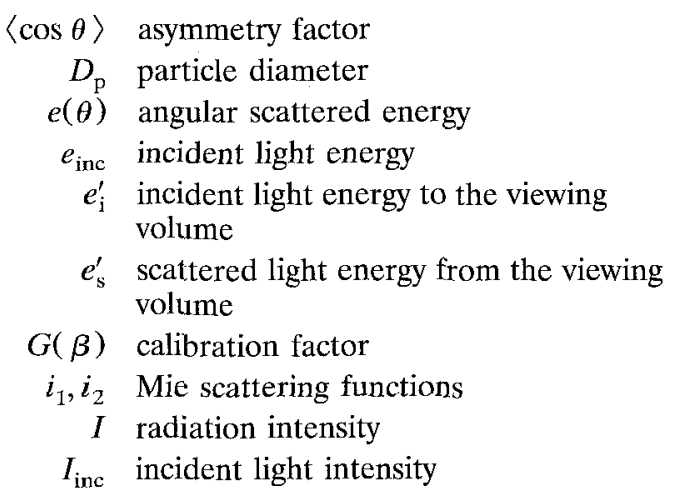

$I_{\mathrm{i}}^{\prime} \quad$ incident light intensity to the viewing volume

$I_{\mathrm{s}}^{\prime} \quad$ scattered light intensity from the viewing volume

$L$ medium length

$m_{\mathrm{g}}$ refractive index of glass

$m_{\mathrm{p}}$ complex refractive index of particle

$m_{\mathrm{w}}$ refractive index of water

$N$ number concentration of particles

$P(\theta)$ phase function

$Q_{\text {ext }}$ extinction efficiency

$Q_{\text {sca }}$ scattering efficiency

$T$ transmittance $=e\left(0^{\circ}\right) / e_{\text {inc }}$

$X$ particle size parameter $=\pi D_{\mathrm{p}} / \lambda$

$\beta$ extinction coefficient

$\lambda$ wavelength in the surrounding medium $=\lambda_{0} / m_{\mathrm{w}}$

$\theta$ scattering angle

$\rho_{\mathrm{o}}$ combined reflectivity of Eq. $6 \mathrm{c}$.

$\sigma$ scattering coefficient

$\tau$ optical thickness $=\beta L$

\section{Subscripts}

E experimental value

M Mie theory results

\section{REFERENCES}

Bangs, L. B. (1987). Uniform Latex Particles. Seradyn, Indianapolis, IN.

Bartholdi, M., Salzman, G. C., Hiebert, R. D., and Kerker, M. (1980). Appl. Opt. 19:1573-1581.

Bohren, C. F., and Huffman, D. R. (1983). Absorption and Scattering of Light by Small Particles. Wiley, New York.

Boll, R. H., and Sliepcevich, C. M. (1956). J. Opt. Soc. Am. 46:200-208

Chae, S. K. (1991). Light Scattering Applications for Microcontamination Control and Radiative Transport Properties Determination. Ph.D. thesis, University of Minnesota, Minneapolis, $\mathrm{MN}$.

Chae, S. K., and Lee, H. S. (1993). Aerosol Sci. Technol. 17:000-000.

Drolen, B. L. (1986). Radiative Transfer with Scattering from Closely-Spaced Spheres. Ph,D. thesis, University of California, Berkeley, CA.

Drolen, B. L., Kumar, S., and Tien, C. L. (1987). AIAA-87-1487, report presented at 22nd Thermophysics Conference. June 8-10, Honolulu, HI. 
Grams, G. W., Dascher, A. J., and Wyman, C. M. (1975). Opt. Eng. 14:85-90.

Grant, D. C. (1988). Sieving Capture of Particles by Microporous Membrane Filtration Media. MS thesis, University of Minnesota, Minneapolis, MN.

Hansen, M. Z., and Evans, W. H. (1980). Appl. Opt. 19:3389-3395.

Holland, A. (1980). Light Scattering by Irregular Shaped Particles. (D. W. Schuerman, ed.). Plenum, New York, pp. 247-254.

Kerker, M. (1969). The Scattering of Light and Other Electromagnetic Radiation. Academic, New York.

Kratohvil, J. P. (1966). J. Colloid Interface Sci. 21:498-512.

Kratohvil, J. P., and Smart, C. (1965). J. Colloid Sci. 20:875-892.

Lanzo, C. D., and Ragsdale, R. G. (1962). Experimental Determination of Spectral and Total Transmissivities of Clouds of Small Particles. NASA Technical Note D-1405, Cleveland, $\mathrm{OH}$.

Lee, H. (1985). Radiation Heat Transfer in Anisotropically Scattering Media. Ph.D. thesis, University of Illinois at Urbana-Champaign, IL.

Love, T. J., and Wheasler, R. A. (1964). An Experimental Study of Infrared Scattering by Clouds of Particles.
ARL 65-110, Aerospace Research Laboratories Dayton, Ohio.

Marshall, T. R., Parmenter, C. S., and Seaver, M. (1976). J. Colloid Interface Sci. 55:624-636.

Menart, J. A. (1988). Experimental Determination of Radiative Properties for Scattering Particulate. MS thesis, University of Illinois at Urbana-Champaign, IL.

Menart, J. A., Lee, H. S, and Buckius, R. O. (1989). Exp. Heat Transfer 2:309-332.

Siegel, R., and Howell, J. R. (1981). Thermal Radiation Heat Transfer. Hemisphere, Washington, DC, pp. 437-438.

van de Hulst, H. C. (1980). Multiple Light Scattering. Academic, New York.

van de Hulst, H. C. (1981). Light Scattering by Small Particles. Dover, New York.

Wagner, P. E. (1985), J. Colloid Interface Sci. 105:456-467.

Wyatt, P. J. (1980). Appl. Opt. 19:975-983.

Wyatt, P. J., and Phillips, D. T. (1972). J. Colloid Interface Sci. 39:125-135.

Received April 2, 1992; accepted October 28, 1992 